\title{
Control of a magnetic Feshbach resonance with laser light
}

\author{
Dominik M. Bauer, Matthias Lettner, Christoph Vo, Gerhard Rempe and Stephan Dürr
}

\begin{abstract}
The capability to tune the strength of the elastic interparticle interaction is crucial for many experiments with ultracold gases. Magnetic Feshbach resonances ${ }^{1,2}$ are widely harnessed for this purpose, but future experiments ${ }^{3-8}$ would benefit from extra flexibility, in particular from the capability to spatially modulate the interaction strength on short length scales. Optical Feshbach resonances ${ }^{9-15}$ do offer this possibility in principle, but in alkali atoms they induce rapid loss of particles due to light-induced inelastic collisions. Here, we report experiments that demonstrate that light near-resonant with a molecular bound-to-bound transition in ${ }^{87} \mathbf{R b}$ can be used to shift the magnetic field at which a magnetic Feshbach resonance occurs. This enables us to tune the interaction strength with laser light, but with considerably less loss than using an optical Feshbach resonance.
\end{abstract}

Using light to change the $s$-wave scattering length $a$ in ultracold gases offers more flexibility than a magnetic Feshbach resonance because it is possible to apply an almost arbitrary spatial pattern of light using holographic masks. The light intensity can vary on a length scale of typically one optical wavelength and the pattern can also be varied rapidly in time. This could be used for a variety of applications, such as the simulation of the physics of black holes ${ }^{3,4}$, the controlled creation of solitons ${ }^{5}$, studies of the collapse of a Bose-Einstein condensate (BEC) in an unusual regime $^{6}$ and the simulation of certain Hamiltonians in which the scattering length needs to be different at different sites of an optical lattice $\mathrm{e}^{7,8}$. Moreover, if each lattice site contains exactly two atoms ${ }^{16}$ and $a$ is varied only on every second lattice site, one could associate molecules at every second lattice site by ramping the magnetic field across the Feshbach resonance, thus producing a quantum state that resembles that of a supersolid. Another possible application for the manipulation of $a$ with light exists in gases consisting of a mixture of different species or spin states. It would be desirable to tune the various scattering lengths in such systems independently, but for that purpose more control parameters than just the magnetic field are needed. Furthermore, if a spatially random light intensity pattern is applied, the scattering length would vary randomly with position, which might give rise to new quantum phases of the atomic gas.

A known scheme to manipulate $a$ using light uses a photoassociation resonance, sometimes also called an optical Feshbach resonance. But so far, photoassociation resonances have rarely been used to tune $a$ because they induce rapid loss of particles. The experiments in refs 12,13 both demonstrated a change of $\operatorname{Re}(a) / a_{\mathrm{bg}}-1 \sim \pm 1$ in ${ }^{87} \mathrm{Rb}$, where $a_{\mathrm{bg}}$ is the background value of $a$. For these parameters, both experiments incurred losses characterized by a two-body rate coefficient $K_{2}$ with an estimated value of $\sim 10^{-10} \mathrm{~cm}^{3} \mathrm{~s}^{-1}$. Typical densities of the order of $10^{14} \mathrm{~cm}^{-3}$ result in lifetimes of the order of $100 \mu \mathrm{s}$, which is too short for

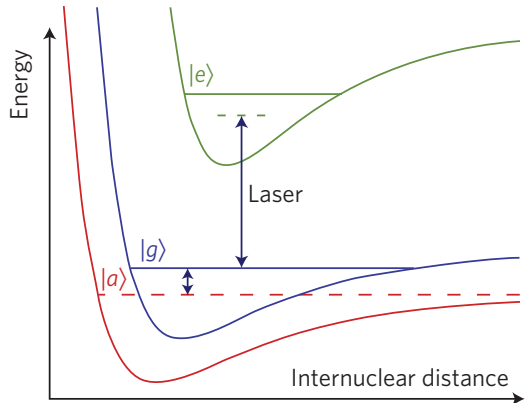

Figure 1 | Level scheme of the experiment. The Feshbach resonance couples atoms at the threshold in the incoming channel $|a\rangle$ to a bound dimer state $|g\rangle$ in a different potential. A laser is near-resonant with a bound-to-bound transition from $|g\rangle$ to an electronically excited dimer state $|e\rangle$.

many applications. Alkaline earth atoms offer the possibility to use photoassociation on narrow intercombination lines, which results in smaller loss rates ${ }^{15}$. But this is not feasible in the large number of experiments with alkali atoms.

Here, we experimentally demonstrate that laser light can noticeably shift a magnetic Feshbach resonance in ${ }^{87} \mathrm{Rb}$ and at the same time induce considerably smaller rates of particle loss than an optical Feshbach resonance. We study the magnetic field dependence of $\operatorname{Re}(a)$ for a large detuning of the laser from a boundto-bound resonance and find a change in $\operatorname{Re}(a)$ similar to that reported for optical Feshbach resonances. Furthermore, we study the system for smaller detunings and observe an Autler-Townes doublet in the particle loss. Our results are in good agreement with a theoretical model.

A basic level-scheme for our experiment is shown in Fig. 1. A light field is near-resonant with a bound-to-bound transition from the dimer state $|g\rangle$ in the electronic ground state to an electronically excited dimer state $|e\rangle$. In our system, the Feshbach resonance coupling between state $|g\rangle$ and the free-atom state $|a\rangle$ is much weaker than the bound-to-bound coupling due to the light field. In the limits of low density or high laser power this becomes true for arbitrary resonances, but for a very broad Feshbach resonance it might be difficult to reach this regime. Furthermore, we use one specific excited state $|e\rangle$ throughout this manuscript, for which the photoassociation coupling between states $|a\rangle$ and $|e\rangle$ is negligible. To understand the physics, it is useful to consider the energy eigenstates that are created from the two dimer states when applying the light. These eigenstates are superpositions of states $|g\rangle$ and $|e\rangle$ with corresponding energy shifts. The Feshbach resonance coupling probes the $|g\rangle$ components of both energy eigenstates, resulting in two resonances as a function of magnetic field $B$, in analogy to an Autler-Townes doublet ${ }^{17}$. 
a

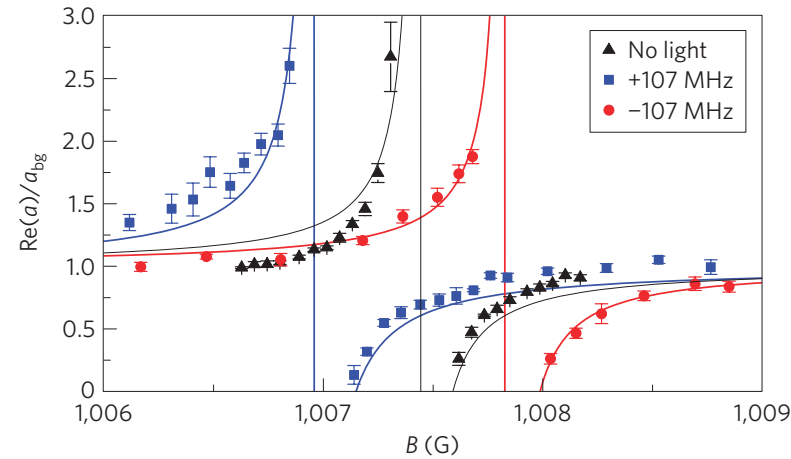

b

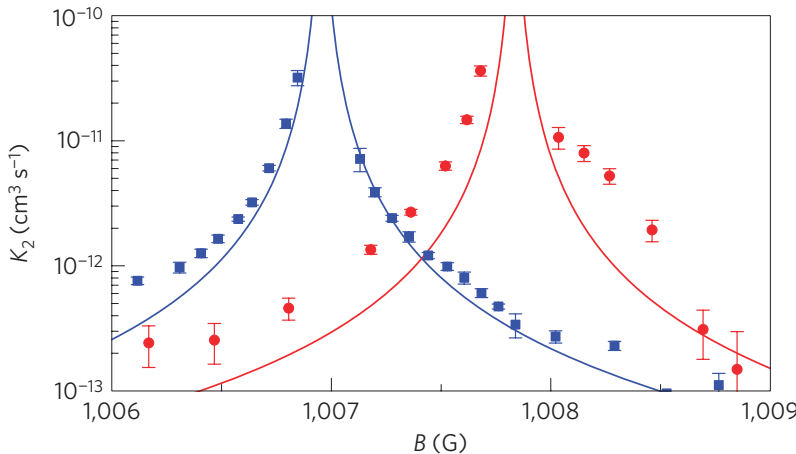

Figure 2 | Shifting a Feshbach resonance with laser light. a, Real part of the scattering length as a function of magnetic field. In the absence of light (triangles), the pole in the scattering length occurs at $1007.4 \mathrm{G}$. With $4.2 \mathrm{~mW}$ of light applied, the Feshbach resonance is shifted to a different magnetic field. The data were recorded at a detuning of the light frequency with respect to the bound-to-bound resonance of $\Delta_{\mathrm{L}} / 2 \pi=+107 \mathrm{MHz}$ (squares) and $-107 \mathrm{MHz}$ (circles). b. The application of the light induces two-body loss described by the rate coefficient $K_{2}$ for a BEC. The observed loss when changing $\operatorname{Re}(a) / a_{\mathrm{bg}}$ by \pm 1 is typically one order of magnitude lower than the loss that would be incurred when using an optical Feshbach resonance. Error bars represent one statistical standard error.

Population in state $|e\rangle$ undergoes spontaneous radiative decay predominantly into other levels, not shown in Fig. 1. This leads to loss of population from the system. With all of the population initially in state $|a\rangle$, this loss can be described by a two-body loss rate coefficient $K_{2}$.

We use a mean-field approach to describe the population in each internal state. We assume that the bound-to-bound resonance and the spontaneous radiative decay both cause faster transitions than the Feshbach resonance coupling so that the population of the states $|g\rangle$ and $|e\rangle$ remains small and can be eliminated adiabatically from the equations of motion. This yields analytic results for the scattering length $a$ and the loss rate coefficient $K_{2}$. More details about the model are discussed in the Methods section. A very similar model was used in ref. 18 to explain an enhancement of
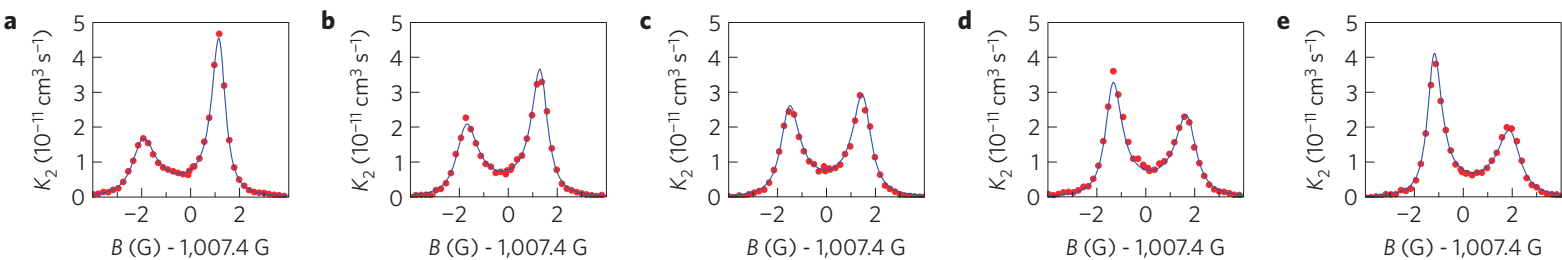

Figure 3 | Autler-Townes splitting of a magnetic Feshbach resonance caused by application of a laser that drives a bound-to-bound transition at a power of $\boldsymbol{P}=\mathbf{0 . 4 7} \mathbf{~ m W}$. a-e, The loss rate coefficient $K_{2}$ is measured as a function of magnetic field $B$ for different values of the laser frequency, which increase from a to e in steps of $1 \mathrm{MHz}$. Part $\mathbf{c}$ is recorded at $382,046,942.62 \mathrm{MHz}$, very close to resonance, where the Autler-Townes doublet becomes symmetric. The lines show a simultaneous fit to all of these data sets. 
a

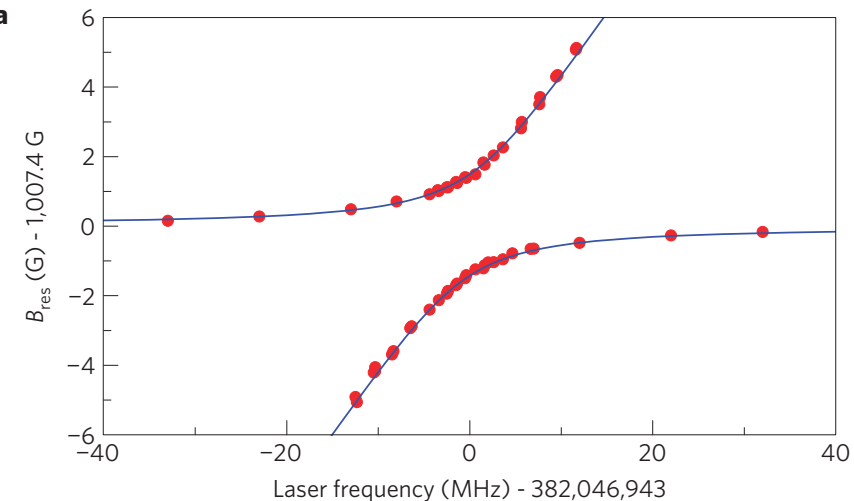

b

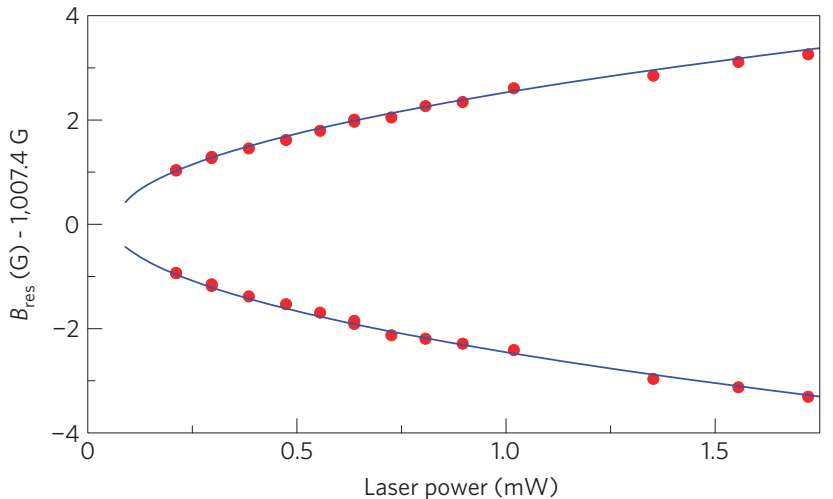

Figure 4 | Systematic study of the resonance positions associated with the Autler-Townes doublet. a,b, The magnetic fields at which the loss resonances occur are shown as a function of laser frequency (a) and laser power (b). Part a was recorded at a power of $0.47 \mathrm{~mW}$ and clearly shows an avoided level crossing. Part $\mathbf{b}$ was recorded at a laser frequency of $382,046,943.00 \mathrm{MHz}$. The solid lines show the prediction of the model in the Methods section with the parameter values obtained from Fig. 3.

light intensity remain at a value far away from $a_{\mathrm{bg}}$. In the second scenario, the magnetic field is held further away from the unshifted Feshbach resonance so that $a \sim a_{\mathrm{bg}}$. Application of the light can shift the Feshbach resonance so close to the chosen value of $B$ that $a$ is modified strongly in regions of high light intensity. In both scenarios, the light causes only a moderate shift of the Feshbach resonance; it does not need to induce a direct photoassociation coupling between the atomic and molecular state, which would result in loss just like for an optical Feshbach resonance.

Not all parameters of our model can be determined from the experiment discussed so far. To do this and to further explore the physics of our system, we study the regime of much smaller detuning of the laser light. In this regime, we clearly observe an Autler-Townes doublet as a function of $B$, as shown in Fig. 3. We fit the model from the Methods section to all of the data in this figure simultaneously. The fit agrees well with the experimental data.

We also recorded the position of the two loss resonances associated with the Autler-Townes doublet systematically as a function of laser frequency and laser power, as shown in Fig. 4. The data show typical features of an Autler-Townes doublet, namely an avoided level crossing in Fig. 4a and a splitting approximately proportional to the square root of the laser power in Fig. $4 \mathrm{~b}$. The data are well described by the model in the Methods section. We conclude that the relevant physics is well understood.

The technique described here could be improved even further by using larger detuning combined with higher laser power, which should reduce the loss. Alternatively, the loss could be reduced by replacing the electronically excited dimer state $|e\rangle$ by a dimer state in the electronic ground state. The coupling to this state could be achieved with a stimulated two-photon Raman transition. Some potential applications of our method were already mentioned in the introduction. A further example is to shift one Feshbach resonance on top of another to explore the coupling between the two. Yet another possible application can be found in ${ }^{133} \mathrm{Cs}$. Its hyperfine state $\left|F=3, m_{\mathrm{F}}=3\right\rangle$ has a very broad Feshbach resonance at a slightly negative magnetic field. Applications of this Feshbach resonance are hampered by rapid loss due to dipolar relaxation at negative magnetic field. Shifting this Feshbach resonance to a positive magnetic field would make it accessible for experiments, such as studies of Efimov physics ${ }^{25}$.

\section{Methods}

A theoretical model for the experiment can be developed as follows. We denote the atomic state as $|a\rangle$, the dimer ground state as $|g\rangle$ and the electronically excited dimer state as $|e\rangle$. We describe the populations of the states with the mean fields $\psi_{\mathrm{a}}, \psi_{\mathrm{g}}$ and $\psi_{\mathrm{e}}$. Generalizing ref. 26, we use the following equations (similar to ref. 18)

$$
\begin{gathered}
i \frac{\mathrm{d}}{\mathrm{d} t} \psi_{\mathrm{a}}=\frac{U_{\mathrm{bg}}}{\hbar}\left|\psi_{\mathrm{a}}\right|^{2} \psi_{\mathrm{a}}+2 \alpha^{*} \psi_{\mathrm{a}}^{*} \psi_{\mathrm{g}}+2 \beta^{*} \psi_{\mathrm{a}}^{*} \psi_{\mathrm{e}} \\
i \frac{\mathrm{d}}{\mathrm{d} t} \psi_{\mathrm{g}}=\alpha \psi_{\mathrm{a}}^{2}+\Delta_{\mathrm{g}} \psi_{\mathrm{g}}+\frac{1}{2} \Omega_{\mathrm{R}}^{*} \psi_{\mathrm{e}} \\
i \frac{\mathrm{d}}{\mathrm{d} t} \psi_{\mathrm{e}}=\beta \psi_{\mathrm{a}}^{2}+\frac{1}{2} \Omega_{\mathrm{R}} \psi_{\mathrm{g}}+\Delta_{\mathrm{e}} \psi_{\mathrm{e}}-\frac{i}{2} \gamma_{\mathrm{e}} \psi_{\mathrm{e}}
\end{gathered}
$$

$U_{\mathrm{bg}}=4 \pi \hbar^{2} a_{\mathrm{bg}} / m$ describes the atomic mean-field energy far away from the Feshbach resonance, where $m$ is the mass of one atom and $\hbar$ is the reduced Planck constant. The electric field of the light is $E=-E_{0} \cos \left(\omega_{L} t\right)$ with amplitude $E_{0}$ and angular frequency $\omega_{L}$. This field causes a coupling on the bound-to-bound transition $|g\rangle \leftrightarrow|e\rangle$. This coupling is treated in an interaction picture with a rotating wave approximation. The corresponding Rabi frequency is $\Omega_{\mathrm{R}}=d_{\mathrm{eg}} E_{0} / \hbar$, where $d_{\mathrm{eg}}=\langle e|d| g\rangle$ is the matrix element of the electric dipole moment. $\gamma_{\mathrm{e}}$ describes spontaneous radiative decay of state $|e\rangle$ into other states, which are not included in the model. Collisional decay is neglected.

The internal energy of each state depends nonlinearly on the magnetic field $B$. Near the pole of the unshifted Feshbach resonance $B_{\text {pole }}$ we approximate this dependence as linear and obtain the internal energies $E_{j}^{\text {int }}=-\mu_{j}\left(B-B_{\text {pole }}\right)+\hbar \omega_{\text {eg }} \delta_{e j}$ for $j \in\{a, g, e\}$ with the magnetic moments $\mu_{j}$ and the Kronecker symbol $\delta_{i j}$. At the Feshbach resonance $\left(B=B_{\text {pole }}\right)$, states $|a\rangle$ and $|g\rangle$ are degenerate, whereas state $|e\rangle$ is offset by an energy $\hbar \omega_{\text {eg }}$. The internal energies are represented in an interaction picture that makes $E_{\mathrm{a}}^{\text {int }}$ vanish and yields $\Delta_{\mathrm{g}}=\mu_{a g}\left(B-B_{\text {pole }}\right) / \hbar$ and $\Delta_{\mathrm{e}}=-\Delta_{L}+\mu_{\mathrm{ae}}\left(B-B_{\text {pole }}\right) / \hbar$ with $\mu_{a g}=2 \mu_{\mathrm{a}}-\mu_{\mathrm{g}}, \mu_{\mathrm{ae}}=2 \mu_{\mathrm{a}}-\mu_{\mathrm{e}}$ and $\Delta_{L}=\omega_{L}-\omega_{\text {eg }}$.

The parameter $\alpha$ with $|\alpha|^{2}=U_{\mathrm{bg}} \mu_{a g} \Delta B / 2 \hbar^{2}$ describes the Feshbach resonance coupling between states $|a\rangle$ and $|g\rangle$, where $\Delta B$ is the width of the Feshbach resonance. The parameter $\beta \propto E_{0}$ describes photoassociation from state $|a\rangle$ to $|e\rangle$, which turns out to be negligible for the parameters of our experiment.

We assume that states $|g\rangle$ and $|e\rangle$ never obtain a large population because $\alpha \psi_{\mathrm{a}}$ and $\beta \psi_{\mathrm{a}}$ are both small compared with $\gamma_{\mathrm{e}}$ and $\Omega_{\mathrm{R}}$. Hence, we can adiabatically eliminate states $|g\rangle$ and $|e\rangle$ and obtain a complex-valued scattering length in analogy to ref. 27

$$
a=a_{\mathrm{bg}}+\frac{m}{2 \pi \hbar} \times \frac{|\alpha|^{2}\left(\Delta_{\mathrm{e}}-i \gamma_{\mathrm{e}} / 2\right)+|\beta|^{2} \Delta_{\mathrm{g}}-\operatorname{Re}\left(\alpha^{*} \Omega_{\mathrm{R}}^{*} \beta\right)}{\left|\Omega_{\mathrm{R}} / 2\right|^{2}-\Delta_{\mathrm{g}}\left(\Delta_{\mathrm{e}}-i \gamma_{\mathrm{e}} / 2\right)}
$$

The two-body loss rate coefficient for a BEC is $K_{2}=-8 \pi \hbar \operatorname{Im}(a) / m$. The Feshbach resonance used in our experiment has the following parameters ${ }^{23,28,29}$ : $B_{\text {pole }}=1007.4 \mathrm{G}, a_{\mathrm{bg}}=100.5 a_{0}, \mu_{\mathrm{a}} / 2 \pi \hbar=1.02 \mathrm{MHz} \mathrm{G}^{-1}, \mu_{\mathrm{ag}} / 2 \pi \hbar=3.8 \mathrm{MHz} \mathrm{G}^{-1}$ and $\Delta B=0.21 \mathrm{G}$. A fit to the data in Fig. 3 yields the following best-fit values for the parameters of the bound-to-bound transition: $\omega_{\mathrm{eg}} / 2 \pi=382,046,942.8 \pm 0.3 \mathrm{MHz}$, $\left|d_{\mathrm{eg}}\right| / e a_{0}=0.28 \pm 0.05, \gamma_{\mathrm{e}} / 2 \pi=4.7 \pm 0.5 \mathrm{MHz}, \mu_{\mathrm{ae}} / 2 \pi \hbar=2.6 \pm 0.1 \mathrm{MHz} \mathrm{G}^{-1}$ and a negligible result for $\beta$. Here, $a_{0}=52.92 \mathrm{pm}$ is the Bohr radius and $e=1.602 \times 10^{-19} \mathrm{C}$ is the elementary charge. The lines in Fig. $4 \mathrm{~b}$ are plotted for $\left|d_{\mathrm{eg}}\right| / e a_{0}=0.31$. We attribute the deviation from the previous value of $d_{\mathrm{eg}}$ to a technical problem with the light intensity calibration. The excited state used here is a specific hyperfine and magnetic substate of the vibrational state $v=120$ in the attractive $1_{\mathrm{g}}$ potential that is adiabatically connected to the ${ }^{2} P_{3 / 2}+{ }^{2} S_{1 / 2}$ threshold (C.-C. Tsai and D. Heinzen, personal communication). 
Received 16 October 2008; accepted 19 February 2009; published online 6 April 2009

\section{References}

1. Tiesinga, E., Verhaar, B. J. \& Stoof, H. T. C. Threshold and resonance phenomena in ultracold ground-state collisions. Phys. Rev. A 47, 4114-4122 (1993).

2. Inouye, S., Andrews, M. R., Stenger, J., Miesner, H.-J., Stamper-Kurn, D. M. \& Ketterle, W. Observation of Feshbach resonances in a Bose-Einstein condensate. Nature 392, 151-154 (1998).

3. Garay, L. J., Anglin, J. R., Cirac, J. I. \& Zoller, P. Sonic analog of gravitational black holes in Bose-Einstein condensates. Phys. Rev. Lett. 85, 4643-4647 (2000)

4. Carusotto, I., Fagnocchi, S., Recati, A., Balbinot, R. \& Fabbri, A. Numerical observation of Hawking radiation from acoustic black holes in atomic Bose-Einstein condensates. New J. Phys. 10, 103001 (2008).

5. Rodas-Verde, M. I., Michinel, H. \& Pérez-García, V. M. Controllable soliton emission from a Bose-Einstein condensate. Phys. Rev. Lett. 95, 153903 (2005).

6. Dong, G., Hu, B. \& Lu, W. Ground-state properties of a Bose-Einstein condensate tuned by a far-off-resonant optical field. Phys. Rev. A 74, 063601 (2006).

7. Abdullaev, F. K., Gammal, A., Salerno, M. \& Tomio, L. Localized modes of binary mixtures of Bose-Einstein condensates in nonlinear optical lattices. Phys. Rev. A 77, 023615 (2008).

8. Deng, X.-L., Porras, D. \& Cirac, J. I. Quantum phases of interacting phonons in ion traps. Phys. Rev. A 77, 033403 (2008).

9. Fedichev, P. O., Kagan, Y., Shlyapnikov, G. V. \& Walraven, J. T. M. Influence of nearly resonant light on the scattering length in low-temperature atomic gases. Phys. Rev. Lett. 77, 2913-2916 (1996)

10. Bohn, J. L. \& Julienne, P. S. Prospects for influencing scattering lengths with far-off-resonant light. Phys. Rev. A 56, 1486-1491 (1997).

11. Fatemi, F. K., Jones, K. M. \& Lett, P. D. Observation of optically induced Feshbach resonances in collisions of cold atoms. Phys. Rev. Lett. 85 4462-4465 (2000)

12. Theis, M. et al. Tuning the scattering length with an optically induced Feshbach resonance. Phys. Rev. Lett. 93, 123001 (2004).

13. Thalhammer, G., Theis, M., Winkler, K., Grimm, R. \& Hecker Denschlag, J. Inducing an optical Feshbach resonance via stimulated Raman coupling. Phys. Rev. A 71, 033403 (2005).

14. Jones, K. M., Tiesinga, E., Lett, P. D. \& Julienne, P. S. Ultracold photoassociation spectroscopy: Long-range molecules and atomic scattering. Rev. Mod. Phys. 78, 483-535 (2006).

15. Enomoto, K., Kasa, K., Kitagawa, M. \& Takahashi, Y. Optical Feshbach resonance using the intercombination transition. Phys. Rev. Lett. 101, 203201 (2008)
16. Volz, T. et al. Preparation of a quantum state with one molecule at each site of an optical lattice. Nature Phys. 2, 692-695 (2006).

17. Autler, S. H. \& Townes, C. H. Stark effect in rapidly varying fields. Phys. Rev. 100, 703-722 (1955).

18. Mackie, M., Fenty, M., Savage, D. \& Kesselman, J. Cross-molecular coupling in combined photoassociation and Feshbach resonances. Phys. Rev. Lett. 101, 040401 (2008).

19. van Abeelen, F. A., Heinzen, D. J. \& Verhaar, B. J. Photoassociation as a probe of Feshbach resonances in cold-atom scattering. Phys. Rev. A 57, R4102-R4105 (1998)

20. Junker, M. et al. Photoassociation of a Bose-Einstein condensate near a Feshbach resonance. Phys. Rev. Lett. 101, 060406 (2008).

21. Marte, A. et al. Feshbach resonances in rubidium 87: Precision measurement and analysis. Phys. Rev. Lett. 89, 283202 (2002).

22. Castin, Y. \& Dum, R. Bose-Einstein condensates in time dependent traps. Phys. Rev. Lett. 77, 5315-5319 (1996).

23. Volz, T., Dürr, S., Ernst, S., Marte, A. \& Rempe, G. Characterization of elastic scattering near a Feshbach resonance in ${ }^{87}$ Rb. Phys. Rev. A 68, 010702(R) (2003)

24. Smirne, G. et al. Collisional relaxation of Feshbach molecules and three-body recombination in ${ }^{87} \mathrm{Rb}$ Bose-Einstein condensates. Phys. Rev. A 75, 020702(R) (2007).

25. Kraemer, T. et al. Evidence for Efimov quantum states in an ultracold gas of caesium atoms. Nature 440, 315-318 (2006).

26. Timmermans, E., Tommasini, P., Hussein, M. \& Kerman, A. Feshbach resonances in atomic Bose-Einstein condensates. Phys. Rep. 315 199-230 (1999).

27. van Abeelen, F. A. \& Verhaar, B. J. Time-dependent Feshbach resonance scattering and anomalous decay of a Na Bose-Einstein condensate. Phys. Rev. Lett. 83, 1550-1553 (1999).

28. Dürr, S., Volz, T., Marte, A. \& Rempe, G. Observation of molecules produced from a Bose-Einstein condensate. Phys. Rev. Lett. 92, 020406 (2004).

29. Dürr, S., Volz, T. \& Rempe, G. Dissociation of ultracold molecules with Feshbach resonances. Phys. Rev. A 70, 031601(R) (2004)

\section{Acknowledgements}

We thank B. Bernhardt and K. Predehl for providing light from their frequency comb. We acknowledge fruitful discussions with T. Bergeman, J. I. Cirac, D. Heinzen, C.-C. Tsai and T. Volz. This work was supported by the German Excellence Initiative through the Nanosystems Initiative Munich and by the Deutsche Forschungsgemeinschaft through SFB 631.

\section{Additional information}

Reprints and permissions information is available online at http://npg.nature.com/ reprintsandpermissions. Correspondence and requests for materials should be addressed to S.D. 